\title{
MECHANISM RESPONSIBLE FOR CHANGES IN THE PUPIL UNACCOMPANIED BY DISTURBANCES OF EXTRA-OCULAR MUSCLE FUNCTION*
}

\author{
BY \\ SYDNEY SUNDERLAND \\ Department of Anatomy and Histology, University of Melbourne, Australia
}

THAT the state of the pupils provides an important clue to lesions developing at the base of the brain has been known for a very long time. There has, however, been little attempt to explain why in some basal lesions pupillary signs precede or develop in the absence of those attributable to paresis or paralysis of the extra-ocular muscles. The object of this paper is to describe one mechanism which may be responsible for the appearance of pupillary signs unaccompanied by impairment of extra-ocular muscle function.

Normal Anatomy.-After emerging from the medial aspect of the cerebral peduncle, the oculomotor nerve passes forwards and laterally to skirt the lateral margin of the posterior clinoid process. The nerve then passes on to, and courses along, the roof of the cavernous sinus for a short distance before entering the lateral wall of that structure. Between the brain stem and the posterior clinoid process the nerve is crossing the interval bounded by the margin of the tentorial notch laterally, the dorsum sellae anteriorly, and the brain stem posteriorly. This interval will be called the tentorial gap.

As it crosses the tentorial gap, the oculomotor nerve has certain important vascular and cerebral relations (Figs 1, 2, 3, and 4). Superior relations are the posterior communicating and posterior cerebral arteries; the latter turns over the nerve on its way laterally while the former is aligned superiorly. The superior cerebellar artery runs beneath the nerve as it travels laterally to pass beneath the tentorium. A portion of the uncal region of the temporal lobe normally extends inwards over the margin of the tentorium and slightly into the tentorial gap; in so doing it becomes closely related, supero-laterally, to the third nerve. It should be emphasized that this portion of the temporal lobe is, in the majority of cases, a normal content of the tentorial gap and a customary superior relation of the third nerve.

An earlier investigation has shown that the pupillo-constrictor fibres are concentrated over the superior arc of the nerve from the cavernous sinus to the mid-brain (Sunderland and Hughes, 1946). Thus two vessels, the posterior cerebral and the posterior communicating, and a portion of the temporal lobe are intimately related to that aspect of the third nerve in which the pupillo-constrictor fibres are concentrated.

* Received for publication July 7, 1952. 

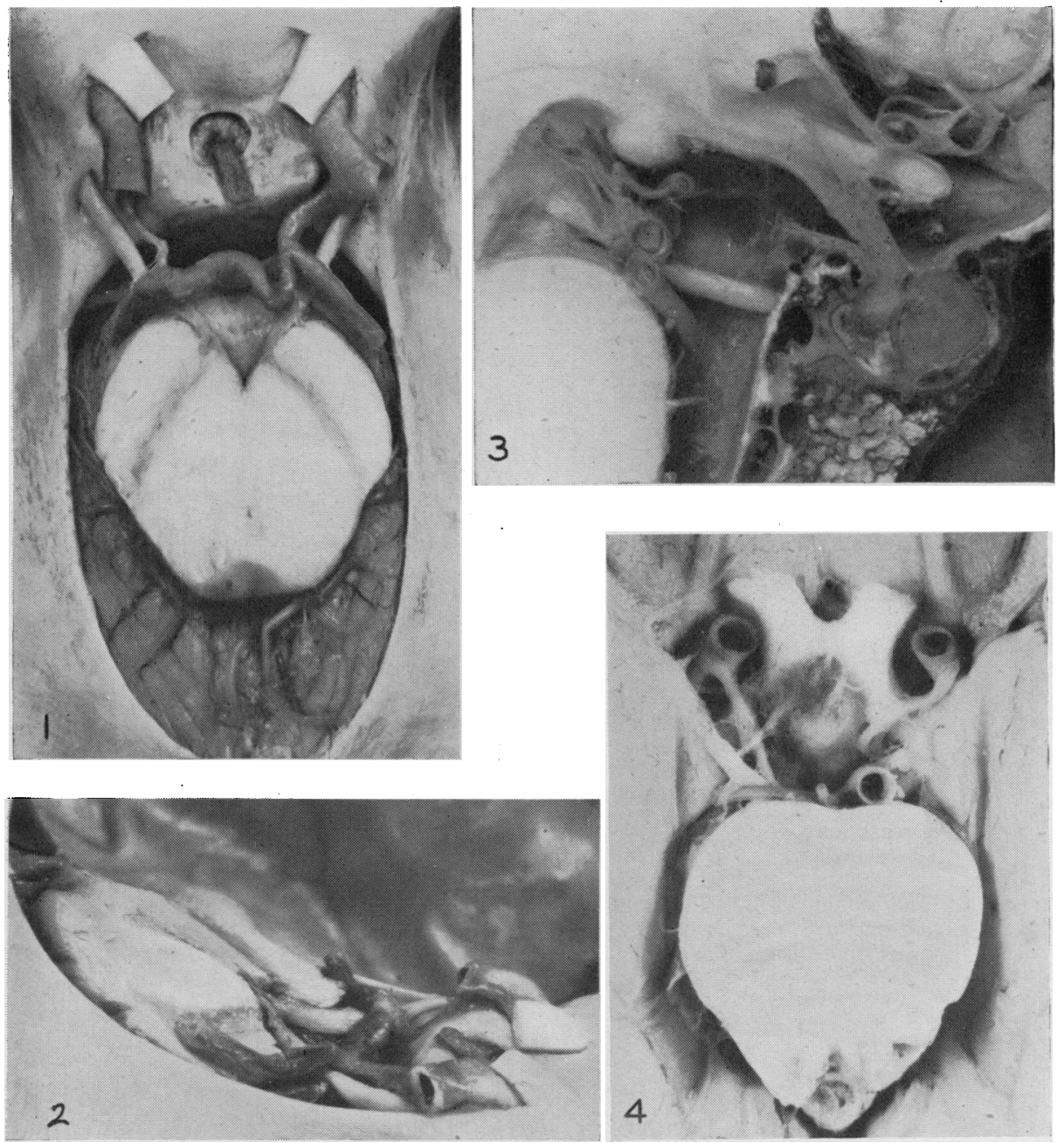

Fig. 1.- Region about pituitary fossa, cavernous sinus, and tentorial notch viewed from above, showing the manner in which the third nerve is related to the posterior cerebral artery, the posterior communicating artery, and the roof of the cavernous sinus.

FIG. 2.-Region illustrated in Fig. 1 viewed from lateral aspect, demonstrating the course of the third nerve and the manner in which it passes between the posterior cerebral and superior cerebellar arteries.

FIG. 3.-Mid-sagittal section of base of brain and base of skull, illustrating the manner in which the third nerve passes between the posterior cerebral and superior cerebellar arteries.

FIG. 4.-Basal aspect of brain illustrating boundaries and contents of interpeduncular fossa. The stump of the third nerve is seen directed outwards and forwards between the posterior cerebral and superior cerebellar arteries. The posterior communicating artery is shown above and medial to the nerve. The indentation made in the temporal lobe by the free margin of the tentorium is clearly shown, as well as that portion of the uncus which bulges over the tentorial margin into the tentorial gap. The third nerve is seen to be a direct inferior relation of the uncal bulge as it passes forwards and outwards. 

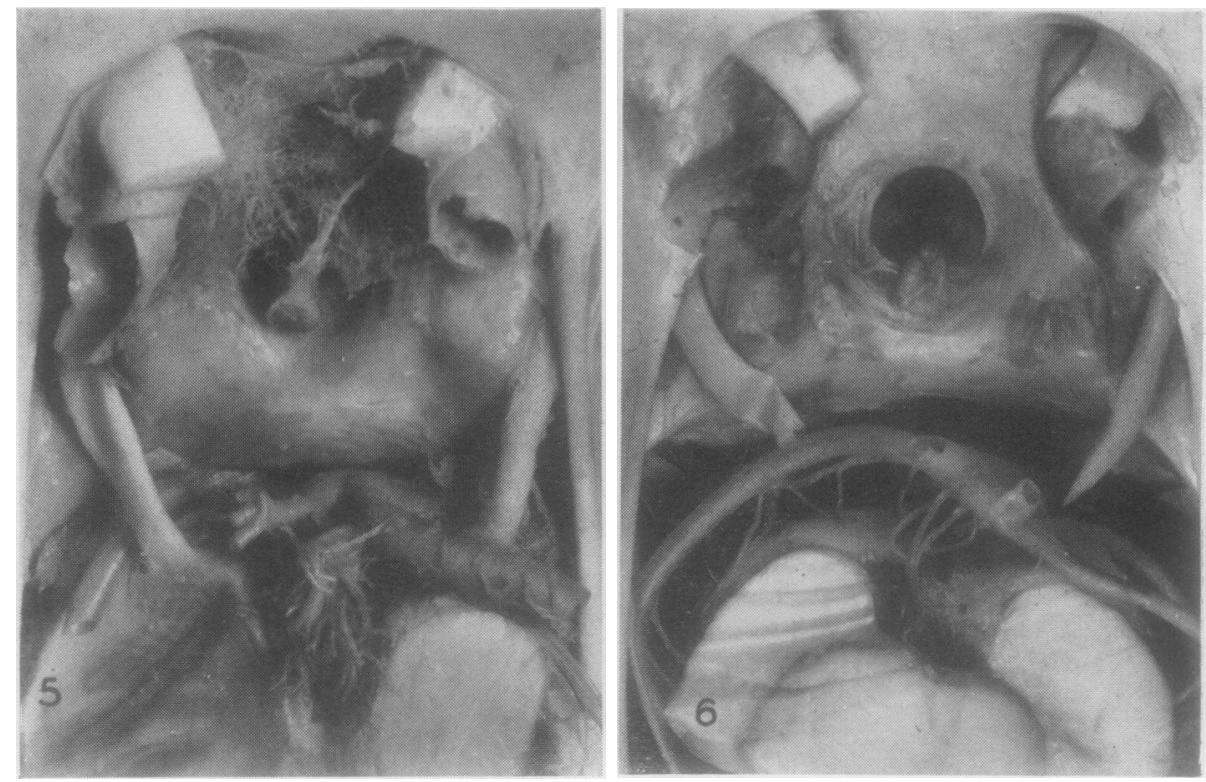

Fig. 5.-Base of skull in region about pituitary fossa and cavernous sinus (from above). The left posterior cerebral artery, which branched from the internal carotid artery, has been removed to reveal the groove outlined by it in the upper surface of the oculomotor nerve.

Fig. 6.--Region about pituitary fossa, cavernous sinus, and interval between brain stem and dorsum sellae (from above). The posterior cerebral artery has been removed on the left side, exposing a groove in the upper surface of the third nerve which was caused by the artery as it crossed and dragged on the nerve.

\section{Anomalous Neuro-Vascular Relation and Aneurysms}

In some individuals the posterior communicating artery establishes contact with the upper surface of the oculomotor nerve and may press upon it. This is particularly likely to occur when this vessel is enlarged to replace the posterior cerebral artery, which then takes origin from the internal carotid artery. Occasionally the pressure exerted by such a vessel may be sufficient to lead to the formation of a groove in the upper surface of the nerve which lodges the vessel (Fig. 5).

Again, the basilar artery may divide into the posterior cerebral arteries at a lower level than usual, or the oculomotor nerves may leave the midbrain at a higher level with reference to the posterior clinoid process. When this arrangement obtains, the vessels ascend more steeply in order to cross the oculomotor nerves on their way to a supratentorial position, and each vessel becomes hooked over the corresponding nerve. Under these circumstances the vessel may drag upon the nerve and on occasions the tension may be sufficient to groove its upper surface (Fig. 6). 


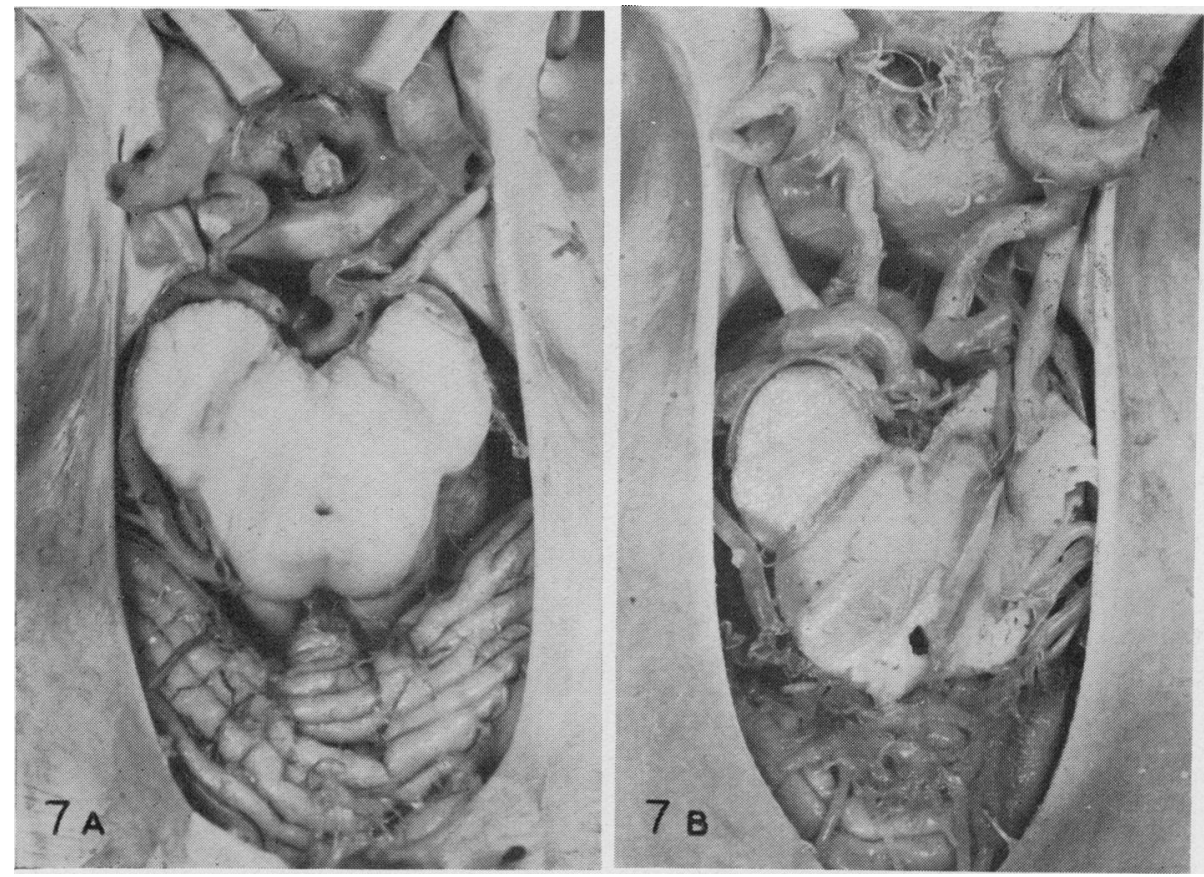

FIG. 7.- Region about pituitary fossa, cavernous sinus, and interval between brain stem and dorsum sellae (from above).

(A) The left internal carotid artery is shown pressing on the oculomotor nerve where that structure is coursing on the roof of the sinus preparatory to entering it.

(B) The right internal carotid artery and the posterior cerebral artery arising from it are shown resting on the oculomotor nerve. The nerve was torn from its origin at the mid-brain during the removal of the brain.

The terminal portion of the internal carotid artery may also rest upon the upper surface of the nerve at the site where the latter is making a delayed entry into the lateral wall of the cavernous sinus. This is most likely to occur when the main trunk of the carotid runs backwards and outwards for a short distance after leaving the sinus and before dividing into its terminal branches (Figs 1 and $7 \mathrm{~A}$ and $\mathrm{B}$ ).

A consideration of these anatomical features makes it clear that the superior surface of the nerve suffers first from pressure exerted by these vessels, and, since the effects are initially confined to that section of the nerve in which the pupillo-constrictor fibres are concentrated, the isolated appearance of pupillary signs is to be expected. Furthermore, under these conditions the nerve may be subjected to intermittent pressure, in which case the pupils will be subject to transient changes.

Finally, aneurysms favour the vessels which are so intimately related to the upper surface of the nerve. They consequently develop above the nerve, and may, as they expand, compress its superior surface and the parasympathetic fibres. In this way they can be responsible for the production 
of signs which would, in the initial stages of their development, be confined to the pupil.

Therefore, in a patient presenting with a dilated pupil and no other signs or symptoms to account for the condition, consideration should be given to the possibility that pressure from an anomalous or arteriosclerotic internal carotid, posterior cerebral, or posterior communicating artery may be an aetiological factor. If an aneurysm is the responsible agent, then as enlargement occurs signs of disturbed extra-ocular muscle function will ultimately be superimposed on those shown in the pupil.

\section{Tentorial Herniation}

Herniations of cerebral tissue through the tentorial notch develop as the result of a wide variety of expanding supratentorial lesions. The cerebral tissue involved is that portion of the temporal lobe which is normally related to the tentorial gap (Fig. 4). Though several accounts are available of the manner in which tentorial herniations develop in a variety of supratentorial expanding lesions, few of these refer to or are concerned with involvement of the third nerve, and those that do, omit significant details pertaining to the mechanism of this involvement and to the associated disturbances of function (Meyer, 1920; Kernohan and Woltman, 1929; Spatz and Stroescu, 1934; Vincent, David, and Thiébaut, 1936; van Gehuchten, 1937; Jefferson, 1938; Schwarz and Rosner, 1941; Evans and Scheinker, 1943; Scheinker, 1945). Exceptions in this respect are the accounts by Dott (1939) and by Reid and Cone (1939).

On the side of the lesion the hernia is, in the initial stages, forced on to the upper surface of the third nerve which is crossing the gap beneath it, while the posterior cerebral artery, which is intervening between the two, deforms both and may subsequently be obliterated between them (Figs 8A and B). The superior portion of the nerve is the first to be affected, and since the pupilloconstrictor fibres are concentrated in this region early changes appear in the pupil on the affected side. These take the form either of an initial brief constriction followed by dilatation, as the pupillo-constrictor fibres are first irritated and later paralysed, or of a dilatation in the absence of any preliminary and transient constriction. Further complications develop as the supratentorial pressure increases. These have been detailed elsewhere (Sunderland and Bradley, 1952); in general they collectively result in the involvement of the remaining fibres of the oculomotor nerve and consequently in the appearance of disturbances in the function of the extra-ocular muscles.

Whether or not pupillary signs appear under these conditions depends on the rate at which the mass of herniated cerebral tissue increases in size and the nerve is distorted. Thus the herniation may develop so slowly that the nerve has time to accommodate itself to the changed conditions, so that persistent disturbance of conduction only occurs when the condition is well established, though transient pupillary changes may occur during the 

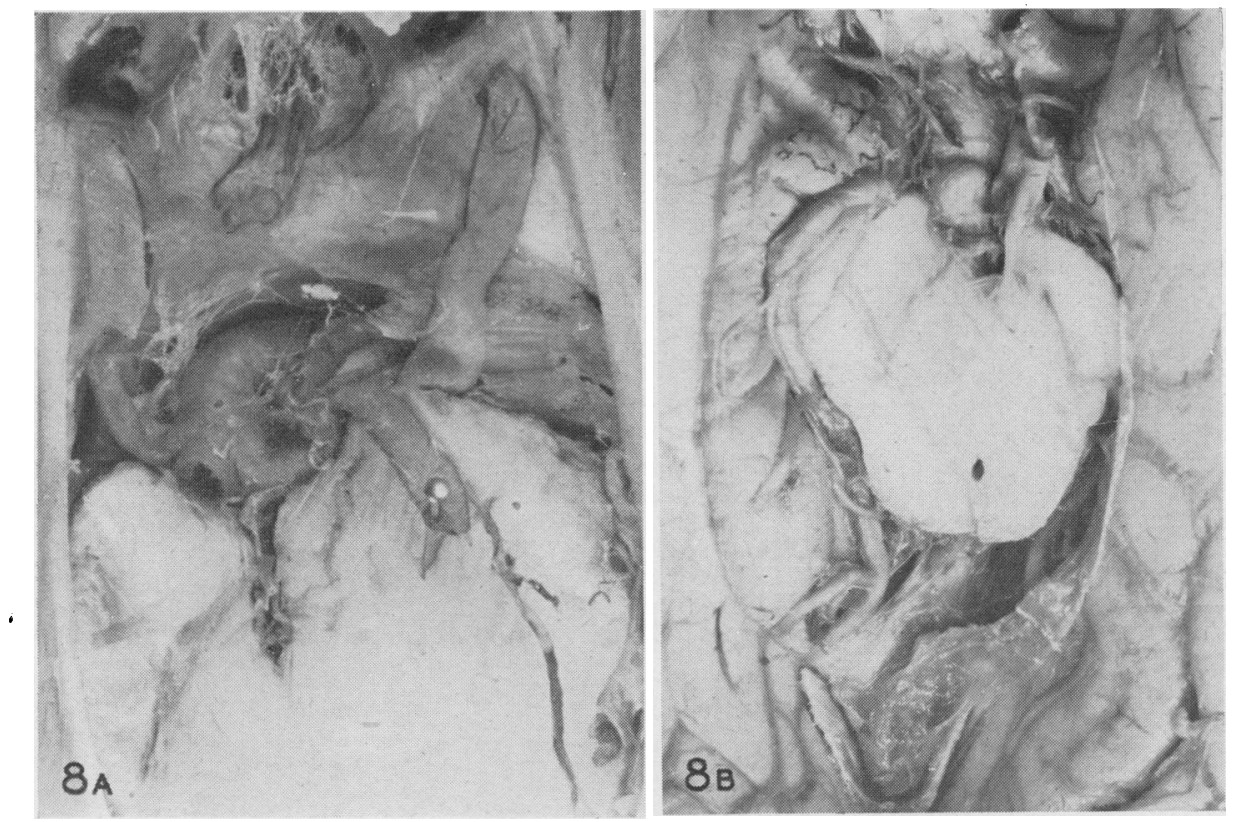

FIG. 8(A).-Region of tentorial notch as seen from above after removal of brain in a case of tentorial herniation. The specimen illustrates the manner in which the posterior cerebral artery has been forced on to the upper surface of the underlying third nerve: The artery has been reflected in order to expose the impression left on the upper surface of the nerve.

FIG. 8(B).-Basal view of brain in a case of tentorial herniation. On the under surface of the anterior portion of the herniation is shown the deep groove outlined and occupied by the third nerve as the herniated tissue was forced down upon it. These post-mortem specimens demonstrate that the superior surface of the third nerve suffers first as the hernia develops and enlarges.

process of accommodation. On the other hand, pupillary changes appear early when the hernia develops rapidly as in certain types of intracranial haemorrhage.

\section{Summary}

(1) Attention is directed to certain vascular and cerebral relations of the third cranial nerve where it runs between the mid-brain and the cavernous sinus.

(2) In this region the posterior cerebral and posterior communicating arteries and a portion of the temporal lobe are intimately related to that aspect of the nerve in which the pupillo-constrictor fibres are concentrated.

(3) Reference is made to the manner in which certain anomalous neurovascular relations, aneurysms, and tentorial herniations may first affect the upper surface of the nerve and in this way produce pupillary changes unaccompanied by any impairment of extra-ocular muscle function. Such 
changes may be intermittent or persistent, depending on the nature of the pathology present. If the lesion is a progressive one, signs of disturbed extra-ocular muscle function are ultimately superimposed on those shown in the pupil.

\section{REFERENCES}

Dotr, N. M. (1939). In Thomson and Miles' “ Manual of Surgery ”, 9th ed., chap. 34., pp. 701-2. ed. A. Miles and D. Wilkie. Oxford University Press, London.

Evans, J. P., and Scheinker, I. M. (1943). Arch. Neurol. Psychiat., Chicago, 50, 258.

VAN GEHUCHTEN, P. (1937). Encéphale, 32, (2), 113.

Jefrerson, G. (1938). Arch. Neurol. Psychiat., Chicago, 40, 857.

Kernohan, J. W., and Woltman, H. W. (1929). Ibid., 21, 274.

MEYER, A. (1920). I Ibid., 4, 387.

REID, W. L., and CoNE, W. V. (1939). J. Amer. med. Ass., 112, 2030.

SCheINKer, I. M. (1945). Arch. Neurol. Psychiat., Chicago, 53, 289.

SCHWARZ, G. A., and RosNer, A. A. (1941). Ibid., 46, 297.

SPATZ, H., and STroescu, G. J. (1934). Nervenarzt, 7, 425, 481.

Sunderland, S., and BradLeY, K. C. (1953). J. Neurol. Neurosurg. Psychiat., 16. In the press. and Hughes, E. S. R. (1946). Brain, 69, 301.

Vincent, C., David, M., and Thíbaut, F. (1936). Rev. neurol., Paris, $65,536$. 